\title{
Sport organizations: An investigation of digital technologies and their impact on performance in the Italian context
}

\author{
Luisa Varriale ${ }^{1, *}$, Paola Briganti $^{1}$, Tiziana Volpe $^{2}$, and Maria Ferrara ${ }^{3}$ \\ ${ }^{1}$ University of Naples "Parthenope", Department of Sport Science and Wellness, Italy \\ ${ }^{2}$ ISMED CNR, Italy \\ ${ }^{3}$ University of Naples "Parthenope", Department of Economics and Business Management, Italy
}

\begin{abstract}
This paper aims to investigate the Italian football clubs outlining their orientation in adopting digital technologies at any level, primarily for improving their overall performance. In the last decades, we observe the increasing implementation and spread of digital technologies worldwide in any economic settings, including the sport industry, where especially in the football business, the digital technologies have been playing a crucial role in supporting managerial, work out and training processes and making them much more competitive and high performing. This study, through a comparative analysis of the Italian football clubs in the Serie A for the year 2021/2022, aims to provide a portrait of these sport organizations and their way to be much more technologically advanced adopting the digital technologies (accessible website; learning and training applications; match analysis tools, etc.). After a brief review of the literature on the topic, we investigate the Italian football clubs mapping them considering some specific main features (i.e. the degree of innovativeness of website, the adoption of e-training or match analysis solutions, etc.). Our qualitative research, through a multiple case study, should provide some possible stimulating and interesting insights for the future, analysing one specific country, where football industry represents the most relevant sport setting with high relevance in terms of volume of affairs, also significantly affected from the digital technologies making them "smart".
\end{abstract}

\section{Introduction}

In the last decades, due to changes derived from technology, individuals have been significantly changed their way to live and work. Some businesses have been changed much more than others, increasingly adopting digital technologies in their different forms, like the sport industry. Indeed, the adoption of Information Technology (IT) and all the digital technologies is remarkably changing and transform all the main aspects of sport

* Corresponding author: luisa.varriale@uniparthenope.it 
organizations, significantly affecting their performance. Sport setting, which traditionally was not recognized as a very relevant industry in terms of economic impact, recently plays a crucial role as significant business and it is characterized by a deep evolution, also due to digital technologies (ICT, social media, AI, IoT, etc.). Thus, the sport field has been characterized from important changes because of technology arousing the increasing interest by scholars and practitioners, who are searching for more effective and efficient tools to manage this business. Indeed, recently, technology has been changed different areas in the sport sector, with relevant social and economic effects.

Starting from the different sub-organizational areas identified in the literature (sport management, sport medicine, athletes' performance improvement, disability and social integration, sporting event management process), this paper focuses on the sport management sub-field, evidencing the main effects derived from the digital technologies within sport organizations that make them "smart". This paper deals with the concept of designing a smart sport organization, that is the investigation of the digital technology to support and make the performance of sport organizations successful. Drawing from previous studies in the literature on the topic, this paper analyzes the main insights and challenges concerning the application of digital technologies within football sport organizations in the Italian context, where the term 'football' is considered as synonymous of the word 'soccer'. We propose to exam in which way digital technologies can affect the performance of the Italian football clubs with emphasis on three main sub-topics: training, management and technical-sport field. Although numerous digital technologies have been introduced in sport, significantly in football sport, in terms of software, technical instruments or digital programs to support the athletes' performance, this phenomenon is still underrepresented in the literature especially with reference to the specific sub-topics and arena analyzed.

After a review of the literature on the topic, this qualitative study, conducted through a multiple case study, aims to provide a portrait of the Italian football organizations, specifically football clubs, identifying and analyzing the main insights and challenges related to the application of digital technologies, specifically innovative digital programs and technical instruments used to improve their performance. Thus, we aim to provide a clear picture of the social, economic and technical implications of digital technologies in the football area with focus on the performance.

\section{Football and technologies: Theoretical background}

In the last two decades, technology improvements in football field let technical staff to analyse in detail the performance of individual players and teams. Moreover, information collected through advanced digital technologies allows coaches and sport scientists to make more easily decisions about the current and future teams' needs, increasing their potential to perform [1]. In particular, the diffusion of match analysis models and recording systems, drone technology and GPS tools has transformed the way to describe, explain, and predict sport performance.

Computerised video tracking systems represent data collection methods to enable sports scientists to identify the current physical demands of players during competition, applying data to program training and testing protocols; additional parameters may be offered by new technologies, such as global or local positioning system technology, allowing technical and tactical factors to complete understanding of physical demands [1].

As highlighted in the main data sources on the topic, such as MEDLINE, SPORTDiscus, PsycINFO, ProQuest, and Teseo, Amisco ${ }^{\circledR}$ and Prozone ${ }^{\circledR}$, Computerised video tracking 
systems are the main advanced technological tools to analyse the physical performance of the elite players. After more than a decade of research on the topic, Castellano and colleagues in their systematic review identify and evaluate the quality of the studies that have utilised semi-automated monitoring systems to quantify the physical profile of outfield players in elite football competitions [1].

Today, elite football teams use expensive and sophisticated semi-automated measurement systems: despite they are 'indirect' means of quantifying physical performance, they provide relevant information on the time-motion characteristics of players, but more development is needed, so additional measures, such as directional changes, jumps, and accelerations, can be quantified accurately [2]. Notwithstanding the numerous advantages, these semi-automated systems still suffer various shortcomings (e.g., cost, variable capture areas and lighting conditions, occlusion between players, and the need for multiple cameras) $[2,3,4]$.

Amisco ${ }^{\circledR}$ was initially used by the French national football team during preparatory matches for the FIFA World Cup Finals in 1998, and subsequently by elite Spanish league teams during the 2002/2003 season [5,6]. The main European competitor, Prozone ${ }^{\circledR}$, was developed during the same period. The validity of the ProzoneTM system was verified by Di Salvo and colleagues $[6,7]$. In the last five years, we also observe a subsequent and continuous increase in the number of publications using such systems. However, experienced analysts are required to code all activities, such as player events (passes, duels, shots, etc.) and set plays (offsides, fouls, corners, etc.) that occur throughout the game. These semi-automated systems can provide useful information about the physical, technical, and tactical activity of players and teams during matches [2], and consequently they have been applied to domestic competitive encounters in the English Premier League, Italian Serie A, Spanish La Liga, French Ligue 1, German Bundesliga, as well as European matches in the UEFA Champions' League and International matches in the UEFA European (UK), which has recently validated these technologically advanced systems $[5,6$, 8].

Researchers have used this technology to determine players' physical profiles in relation to factors such as player position, fatigue or pacing, match half variation, competitive standard, and the influence of contextual variables, such as match location, the quality of the opposition and match status. These variables affect the way in which play unfolds but also the physical demands, their technical performance, the effective playing time, or the percentage ball possession for each team. Recently, this technology has also been used to analyse the dynamics of play by focusing on team surface, length, and width $[9,10]$.

In their research Sarmento and colleagues [11] reviewed the literature on match analysis in football male adult athletes, reaching the same results than match analysis on men football players. They conducted a systematic review collecting contributions from the Institute for Scientific Information (ISI) Web of Knowledge database using PRISMA (Preferred Reporting Items for Systematic reviews and Meta-analyses) guidelines. The following keywords were used: football and soccer, each one associated with the terms: match analysis, performance analysis, notational analysis, game analysis, tactical analysis and patterns of play. They analysed the outcome measures of selected studies, distinguishing between descriptive, comparative or predictive research design: descriptive studies focused on a description of technical, tactical and physical performance variables; comparative studies considered as dependent variables "playing position" and "competitive level"; predictive researches were the few ones, although literature highlighted to pursue this purpose, and focused on modelling football performance, specifically soccer as term. During the last ten years, the evolutionary tendencies of the research on the topic shows that effective situational co-variables of football performance have been identified and 
considered, such as game location, quality of opposing teams, match status and match half, using standardised categories and description of activities and participants, and integrating the situational and interactional contexts into the analysis of football performance.

In particular, the football match performance represents the result of the interaction of different technical, tactical, mental and physiological factors [12, 13]. Despite, matchanalysis has been predominantly done using simple description and associations between variables as described in the research published in scientific journal; this phenomenon could not have been effectively explored without considering the dynamic, interactive and complex systems' aspects that can better characterise match performance in football [14, 15].

Besides match analysis tools, the Drone technology is often used and football teams to record their training of football players and see if they can make any adjustments. Many American Football teams use aerial imagery to research and develop players' techniques and movements: step-by-step team moves are often the difference between winning and losing games [16].

Drones (aerial vehicle) enable high-quality shots and photos to be taken from high altitude. The key benefit of using Drones is that they can easily reach anywhere in the pitch, which is very difficult for coaches to reach. It's likely that for football coaches, position and shape of the team are very hard to see from the technical area. Football coaches and managers have only one side-view of each action in the technical area, which is very narrow and prone to misinterpretation of tactics. The player's position (own and opponent's) and movement of the player's on the pitch are very dynamic. As a result, numerous space creations occurred on the field. The coach needs to systematically analyse these spaces. In the attacking team, players need to stretch and create space to receive the ball and then compact certain spaces immediately after losing the ball to make it harder for the opposition to play. So the position of football players is changing repeatedly. Therefore, one of the greatest tools is to discuss the practical session with the players and to evaluate the positioning review $[17,18]$.

According to football match analyst, "the drone is useful for all types of drills, collective or individual. With the angle of the aerial view, it's easier to work the moments of the game, since fundamental principles as space, distance and, the relation between players are more and better identifiable than with the normal broadcast or side tactical camera angles". Besides, it is noted that some coaches go up into the stand during occasions. In modern football, positional data are needed to understand the tactical performance of players $[19$, 20, 21].

The Drone can keep an eye on the pitch, as well as the ball to track the positional data. Above all, the distribution of players on the pitch during the attack and defence is a key performance metric when evaluating the spatial strategy. In the same way, team dispersion can be evaluated by measuring the length and width of the teams while the ratio of length to width inform about the team's shape. Thus, the bird's eye view angle provided by Drones is giving a wide-angle that can range from end zone to end zone. Powderly [18] found that an overhead Drone camera caught a bird's eye view of the soccer game. He further elaborates, Drone footages might have explained the actual positioning to central midfield. Roberto Martinez, the former Everton's mentor, wants all his tactical sessions filmed utilized drone technology. So, it might have been obvious that modern coaches are now willing to use Drone technology to evaluate their coaching sessions [22].

Recently, Sparks and colleagues [23] have reported an analysis of university-level football players with GPS technology using an individual threshold to categorize the running demands during a match. According to Dwyer and Gabbett [24], these authors 
analysed the low, moderate and high intensity velocity zone of players, and individualized intensity zones with the time spent in different internal zone (heart rate) during competitions [23], differentiating with GPS technology the relative running demands by playing position during a match [25].

Table 1 - Technological tools to analyse the physical performance of the elite players.

\begin{tabular}{|c|c|}
\hline Match analysis models and recording systems & $\begin{array}{l}\text { Computerised video tracking systems } \rightarrow \text { MEDLINE, } \\
\text { SPORTDiscus, PsycINFO, ProQuest, and Teseo, } \\
\text { Amisco } \AA \text { and Prozone } \AA \\
\text { Semi-automated measurement systems } \rightarrow \text { Amisco } \AA \\
\text { and Prozone } \AA \\
\text { Preferred Reporting Items for Systematic reviews and } \\
\text { Meta-analyses } \rightarrow \text { PRISMA guidelines }\end{array}$ \\
\hline Drone technology & $\begin{array}{l}\text { The bird's eye view angle provided by Drones is } \\
\text { giving a wide-angle that can range from end zone to } \\
\text { end zone. }\end{array}$ \\
\hline GPS technology & $\begin{array}{l}\text { Individual threshold to categorize the running demands } \\
\text { during a match. }\end{array}$ \\
\hline
\end{tabular}

Source: Our processing.

\section{Methodology}

The research method for this study consists of the multiple case study. One of the most representative and major professional Football league, that is the Italian Football clubs, has been investigated, analyzing its disclosure sources, mainly website. Indeed, starting from a review of the main contributions of literature and adopting a qualitative methodology, this study provides an in-depth analysis of the technological solutions and initiatives introduced and used by the Italian football clubs. Specifically, the research aim mainly is to investigate the orientation and the degree of innovativeness in terms of adopting digital technologies by teams playing in the Italian Serie A in the 2021/2022 season.

Therefore, this empirical analysis aims to provide a portrait of the Italian football clubs, deepening the behavioural models and initiatives introduced from the perspective of digital technologies and, thus, outlining the degree of technological innovativeness with impact on their performance. In detail, a multiple case study approach [26, 27] is adopting for achieving descriptive purposes. In our research we describe the current state of the degree of technological innovativeness of the Italian football clubs investigated. According to the literature, a qualitative study, because of being a case study, cannot validate theoretical hypotheses or move towards a "grounded theory" [28]. The sample is made up of 20 case studies corresponding to the Italian football clubs in Italian Serie A in the 2021/2022 season.

A manual content analysis of all the documentation available was conducted, specifically primary and secondary data were considered. Information and data about the digital technologies introduced and implemented by the Italian football, in the different fields (training, management and technical-sport) were collected and processed through reports (FIFA report, Istat, etc.), analysis of archival data, football clubs' documentation, football clubs' websites, football clubs' social network sites (SNS), press and magazines, focusing on issues related to digital technologies and performance.

We have identified and investigated all the solutions, programs and actions promoted and introduced by the football clubs analysed to outline their overall degree of technological innovativeness. To complete the analysis, some of the most significant experiences and solutions carried out by some football clubs were compared to identify the 
key organizational and managerial implications that characterize the main differences with a specific focus on their approach and orientation to technological innovativeness and performance.

\section{Results and Discussion}

The sample investigated consists of 20 football clubs in the Italian Serie A in the 2021/2022 season, only three of them listed on the stock exchange. The overall 20 Italian football clubs have a website and use specific applications for providing and accessing to different services; only 10 football clubs use e-learning and e-training solutions, as well as 11 football clubs tend to adopt technological solutions related to match analysis.

Thus, the main lines of solutions and activities promoted and implemented by the football clubs investigated adopting digital technologies can be traced back to three areas of competence: 1) support for football coaches and managers; 2) reception and accommodation desk, ticketing, selling gadgets; 3) learning process for staff (technical, medical and managerial) and football training for athletes and coaches. In these three areas we can observe several technological solutions implemented in order to improve the overall performance making the football clubs much more 'smart'.

The AC Milan Football Club presents a specific application for buying tickets, receiving information about vip hospitality, prices and parking services. Also, the club has its own Milan Academy which consists of the set of projects dedicated to the involvement of an U18 target audience in sporting activities via the football coaching promoted by AC Milan Team and the training of all adults involved also using advanced technological solutions, especially at the time of the Covid-19 pandemic. Furthermore, the Milan Academy is linked to Milan Junior Camp. Indeed, "the training proposal is aimed at national and international sports clubs, both amateur and professional, sports coaches and managers, events clubs, federations, institutions and sponsors who, in various ways, aim to generate a sporting culture and organisational excellence in their territory." (from official website). The Milan Academy, AC Milan's true centre of innovation, is shaped by a team of sports professionals made up of managers, football coaches, teachers and instructors, who operate with knowledge and competence in accordance with the Club's principles and values and also aspire to define a new standard for the world of football, including advanced technologies. "Passion, Excellence, Sophistication and Teamwork are the building blocks of the Project which, by sharing the Milan Method and using guidelines, coaching programmes and dedicated services, supports the adults who follow daily the human and sporting development of all the youngsters involved in football and sporting activities" (from official website). Finally, Milan X-Experience is "dedicated to football clubs, events companies or travel agencies, the Milan X-Experience offers an overview of the Rossoneri [red-black] world, allowing participants to carry out training sessions in AC Milan's facilities for a week as well as discovering everything else that forms AC Milan's world. The groups that participate in the Milan Experience therefore undergo a completely personalised experience: training sessions with the official Milan Academy coaches, joint training sessions with our Football Schools, a visit to Casa Milan, San Siro and other activities on the pitch on Match Day. All this makes for an absolutely unforgettable experience!" Milan Experience provides several further services including: A Milan Academy dedicated account, Milan Academy Coach, Training at AC Milan's facilities, Pre-match activities at San Siro, A visit to and lunch at Milan Home, Museum visit, Puma Kit-Milan Academy, Certificate of participation, Shop online (https://www.acmilan.com/it). 
The Atalanta Football Team presents the following technological areas: academy (for learning and training), shopping, magazine, and ticketing applications, online courses (https://www.atalanta.it/).

The Bologna Football Team presents specific sections for Kids, Ticketing online, Store, Amazon, ESports Academy adopting technological solutions (https://www.bolognafc.it/).

The Cagliari Football Team provides several services supported by technologies, such as ticketing online, super green pass instructions, and academy (www.cagliaricalcio.com).

The Empoli Football Team presents the following technological areas: Ticket, news, kids and teenagers sector, female team, Empoli For Charity Onlus, and Empoli Esports (https://empolifc.com/).

The Fiorentina Football Team concerns the following technological areas: Online store, Podcast, Ticketing online, Photogallery (https://www.acffiorentina.com/it).

The Genoa Football Team regards the following technological areas: Web shop, Genoa chanel, Podcast, Photo gallery, Genoa exports, Charity Genoa and smart working forms for managerial staff, including e-learning solutions (www.genoaacfc.it).

In the Inter Football Team there are the following technological areas: Ticketing online, shopping, Interclub, podcast, Inter TV, virtual museums through virtual tours, fans Token \$INTER, social wall (share your passion for your team and mention the official page @inter), Imscudetto (live again the overall season of your football team - blackblue season: click here I M SCUDETTO STORY) (https://www.inter.it/it).

For the Juventus Football Team the specific technological areas are: Ticketing online for match, visiting Museum\&Allianz Stadium Tour Matchday Tour, web store, Juventus TV, Juventus radio, SMART Black\&White Membership (the digital affiliation program, for all the fans physically far but who love to feel part of the big black-white family without limits, especially geographic limits), Juventus TV pass the entire football season, access to digital contents and events, discounts and promotions and dedicated contests, Fan club, smart working (www.juventus.com).

The Lazio Football Team has the following technological areas: Ticketing online, Fan Zone, WebTv, and Fidelity Area (https://www.sslazio.it/it/).

The Napoli Football Team presents the following technological areas: Fans, Fan Club, web store, ticketing online, video gallery, fotogallery, SSCN charity, and live match (https://www.sscnapoli.it/).

The Roma Football Team has the following technological areas: Shopping, ticketing online, Roma Cares (Charity), fan zone, ESports, and work shop for coaches (https://www.asroma.com/it/).

In the Salernitana Football Team website, there is the fidelity card online procedure for the activation or renewal of the card; also there is the service 'Box to box' inspired to these terms, the stories of the football team players can be shared passing form one bow to another remembering the steps of their career (https://www.ussalernitana1919.it/).

The Sampdoria Football Team has the following technological areas: Academy, ESports, Sampcity, and Ticketing online (website address is: https://www.sampdoria.it/).

In the Sassuolo Football Team we have the following technological areas: Tickets on line, Web Store, Sassuolo channel, photo gallery, whatapp, and club (www.sassuolocalcio.it).

The Spezia Football Team has as technological areas: Spezia channel, Web tickets, photo gallery, video gallery, and whatapp (www.acspezia.com) 
The Torino Football Team includes: Web tickets, Torino channel, photo gallery, web $\mathrm{TV}$, fidelity heart card, Toro life club (www.torinofc.it).

The Udinese Football Team has the following technological areas: Web shop, ticketing online, fan club, Udinese TV, ESports, Monthly Udinese, podcast, Udinese summer camp on line (e-learning) (www.udinese.it).

In the Venezia Football Team there are the following technological areas: Official Webstore and tickets online (https://www.veneziafc.it/).

Finally, the Verona Football Team has the following technological areas: HV channel radio, HV channel, Fidelity card, and ticketing online (https://www.hellasverona.it/).

The analysis conducted, which briefly outlined a summary framework of the Italian football clubs in the Serie A for the 2021/2022 season regarding the topic of technological innovativeness and performance, allows us to make some considerations in order to clarify the degree of innovativeness related to technology of the same football clubs through the use and adoption of digital technologies (see Table 2 for a summary):

1. the majority of football clubs has their own website and use its also primarily for providing some basic services, such as ticketing online and connections to social media (official facebook page or TV channel, tec.) that have policies and measures aimed at promoting inclusion making their environment physically and virtually (website) accessible and usable in practice tend to substantially transpose and apply the current legislation on the protection of weak categories or guarantees for the fragile or in difficulty categories;

2. the football clubs with a greater orientation to the adoption of technologies, such as Juventus or AC Milan, are mostly concentrated in Northern and Central Italy, and they used to be part of Serie A for the last five seasons, showing that the constant position in Serie A also helps to be much more innovative in their management and their orientation to develop and adopt technological solutions is correlated to their high performance;

3. most football clubs with a long history stand out more for the promotion and adoption of technological solutions for being much more smart.

Table 2: A brief portrait of the Italian Serie A Championship in the 2021/2022 season.

\begin{tabular}{|l|c|c|c|c|}
\hline $\begin{array}{c}\text { Soccer Teams } \\
\text { A Serie Italy } \\
\mathbf{2 0 2 1 - 2 0 2 2}\end{array}$ & $\begin{array}{c}\text { Website } \\
\text { (Yes/No) }\end{array}$ & $\begin{array}{c}\text { E-learning/training } \\
\text { solutions (Yes/No) }\end{array}$ & $\begin{array}{c}\text { Match Analysis } \\
\text { (Yes/No) }\end{array}$ & $\begin{array}{c}\text { Applications } \\
\text { (Yes/No) }\end{array}$ \\
\hline AC Milan & $\mathrm{Y}$ & $\mathrm{Y}$ & $\mathrm{Y}$ & $\mathrm{Y}$ \\
\hline Atalanta & $\mathrm{Y}$ & $\mathrm{Y}$ & $\mathrm{Y}$ & $\mathrm{Y}$ \\
\hline Bologna & $\mathrm{Y}$ & $\mathrm{Y}$ & $\mathrm{Y}$ & $\mathrm{Y}$ \\
\hline Cagliari & $\mathrm{Y}$ & $\mathrm{N}$ & $\mathrm{N}$ & $\mathrm{Y}$ \\
\hline Empoli & $\mathrm{Y}$ & $\mathrm{Y}$ & $\mathrm{N}$ & $\mathrm{Y}$ \\
\hline Fiorentina & $\mathrm{Y}$ & $\mathrm{N}$ & $\mathrm{N}$ & $\mathrm{Y}$ \\
\hline Genoa & $\mathrm{Y}$ & $\mathrm{Y}$ & $\mathrm{Y}$ & $\mathrm{Y}$ \\
\hline Inter & $\mathrm{Y}$ & $\mathrm{Y}$ & $\mathrm{N}$ & $\mathrm{Y}$ \\
\hline Juventus & $\mathrm{Y}$ & $\mathrm{Y}$ & $\mathrm{Y}$ & $\mathrm{Y}$ \\
\hline Lazio & $\mathrm{Y}$ & $\mathrm{N}$ & $\mathrm{N}$ & $\mathrm{Y}$ \\
\hline Napoli & $\mathrm{Y}$ & $\mathrm{Y}$ & $\mathrm{N}$ & $\mathrm{Y}$ \\
\hline Roma & $\mathrm{Y}$ & $\mathrm{N}$ & $\mathrm{N}$ & $\mathrm{Y}$ \\
\hline Salernitana & $\mathrm{Y}$ & $\mathrm{N}$ & $\mathrm{Y}$ & $\mathrm{Y}$ \\
\hline Sampdoria & $\mathrm{Y}$ & $\mathrm{Y}$ & $\mathrm{N}$ & $\mathrm{Y}$ \\
\hline Sassuolo & $\mathrm{Y}$ & $\mathrm{N}$ & $\mathrm{Y}$ & $\mathrm{Y}$ \\
\hline Spezia & $\mathrm{Y}$ & $\mathrm{N}$ & $\mathrm{Y}$ & $\mathrm{Y}$ \\
\hline Torino & $\mathrm{Y}$ & $\mathrm{Y}$ & $\mathrm{N}$ & $\mathrm{Y}$ \\
\hline Udinese & $\mathrm{Y}$ & $\mathrm{N}$ & $\mathrm{N}$ & \\
\hline Venezia & $\mathrm{Y}$ & & $\mathrm{Y}$ & \\
\hline Verona & $\mathrm{Y}$ & & & \\
\hline & & & & $\mathrm{N}$ \\
\hline
\end{tabular}

\section{Concluding remarks}

Adopting the perspective of technological innovativeness and organizational performance, this study provides a synthetic analysis of the Italian context by taking a picture of the Italian football clubs. Specifically, this study outlines the relevance to better 
disclose and also create the conditions for sharing and comparing the different experiences and expertise of the football clubs using advanced technologies for supporting their managerial and technical staff, as well as the fans and the overall stakeholders. Indeed, we observe that football clubs present still limited orientation to develop and adopt technological solutions and sharing them appropriately, and also it is clear the need to promote internally at football clubs, involving managers and coaches and athletes, a significant process of change, especially reducing the resistance to the technological change.

Starting from the limitations of this study, especially for its exploratory nature, in the future we aim to conduct some in-depth and semi-structured interviews to Human Resource Managers of Italian football clubs of the Serie A in order to collect specific information and data for identifying exactly the technological tools adopted for human resource recruitment, recruitment and socialization, football training, learning process, career programming, evaluation, and compensation, and also more information about e-learning solutions and etraining programs, as well as advanced technology systems and applications, like match analysis systems. Indeed, information and data could be obtained only through interviews to training managers and technical staff and directly to match analysts within the organizations.

\section{References}

1. J. Castellano, D. Alvarez-Pastor, P. S. Bradley. Evaluation of research using computerised tracking systems (Amisco ${ }^{\circledR}$ and Prozone ${ }^{\circledR}$ ) to analyse physical performance in elite soccer: A systematic review. Sports medicine, 44(5), 701-712 (2014)

2. C. Carling, J. Bloomfield, L. Nelsen. The role of motion analysis in elite soccer: contemporary performance measurement techniques and work rate data. Sports Med.; 38(10): 839-62 (2008)

3. A. Redwood-Brown, W. Cranton, C. Sunderland. Validation of a real-time video analysis system for soccer. Int J Sports Med.; 33: 635-40 (2012)

4. S. Barris, C. Button. A review of vision-based motion analysis in sport. Sports Med.; 38(12):1025-43 (2008)

5. V. Di Salvo, A. Collins, B. McNeill B. Validation of ProZone: a new video-based performance analysis system. Int J Perf Anal Sport.; 6: 108-19 (2006)

6. V. Di Salvo, W. Gregson, G. Atkinson. Analysis of high- intensity activity in Premier League soccer. Int J Sports Med. 2009; 30(3): 205-12 (2009)

7. P. S. Bradley, W. Sheldon, B. Wooster. High-intensity running in English FA Premier League soccer matches. J Sports Sci.; 27(2): 159-68 (2009)

8. A. Dellal, D. P. Wong, W. Moalla. Physical and technical activity of soccer players in the French First League - with special reference to their playing position. Int Sport Med J.; 11(2): 278-90 (2010)

9. R. Bartlett, C. Button, M. Robins. Analysing team coordi- nation patterns from player movement trajectories in soccer: methodological considerations. Int J Perf Anal Sport.; 12: 398-424 (2012)

10. J. B. Taylor, S. D. Mellalieu, N. James. The influence of match location, quality of opposition, and match status on technical performance in professional association 
football. J Sports Sci.; 26(9): 885-95 (2008)

11. H. Sarmento, R. Marcelino, M. T. Anguera, J. CampaniÇo, N. Matos, J. C. LeitÃo. Match analysis in football: a systematic review. Journal of sports sciences, 32(20): 1831-1843 (2014)

12. C. Carling, T. Reilly, A. Williams (Eds.). Performance assessment for field sports. London: Routledge (2009)

13. B. Drust, G. Atkinson, T. Reilly. Future perspectives in the evaluation of the physiological demands of soccer. [Review]. Sports Medicine; 37(9): 783-805 (2007).

14. N. Balague, C. Torrents, R. Hristovski, K. Davids, D. Araújo. Overview of complex systems in sport. Journal of Systems Science \& Complexity; 26(1): 4-13 (2013)

15. J. Sampaio, V. Maçãs. Measuring tactical behaviour in football. International Journal of Sports Medicine; 33(5), 395-401 (2012).

16. M. S. Islam. Introducing drone technology to soccer coaching. Int J Sports Sci Phys Educ; 5(1): 1-4 (2020)

17. Q. Arena. Are Drones the Future of Sport Training? Quadcopter Arena 2018. https://quadcopterarena.com/are-drones-the-future-of-sport-tra ining/ (accessed January 21, 2020).

18. D. Powderly. Drones In Football. Medium 2016. https://medium.com/@David_Powderly/drones-in-football-65_e275681eba (accessed January 21, 2020).

19. R. Rein, D. Memmert. Big data and tactical analysis in elite soccer: future challenges and opportunities for sports science. Springer Plus 2016; 5. https://doi.org/10.1186/s40064-016-3108-2.

20. A. Ric, C. Torrents, B. Gonçalves, L. Torres-Ronda, J. Sampaio, R. Hristovski. Dynamics of tactical behaviour in association football when manipulating players' space of interaction. PLOS ONE 2017; 12: e0180773. https://doi.org/10.1371/journal.pone.0180773.

21. L. Viegas. Drone changing soccer analysis. Soccer HUB 2019. https://hubsoccer.com/2019/07/03/drone-changing-soccer-ana lysis/ (accessed November 21, 2021).

22. P. Kirkbride. Everton FC use drone technology to film training sessions Liverpool Echo 2015. https://www.liverpoolecho.co.uk/sport/football/footballnews/ everton-fc-use-drone-technology-10013696 (accessed November 21, 2021).

23. M. Sparks, B. Coetzee, T. J. Gabbett. Internal And External Match Loads Of University-Level Soccer Players: A Comparison Between Methods. Journal of Strength and Conditioning Research Journal of Strength [Epub ahead of print] (2016)

24. D. B. Dwyer, T. J. Gabbett. Global positioning system data analysis: Velocity ranges and a new definition of sprinting for field sport athletes. The Journal of 
Strength \& Conditioning Research; 26(3): 818-824 (2012)

25. F. J. N. Sánchez, F. J. T. Bendala, M. Á. C. Vázquez, L. J. S. Moreno-Arrones. Individualized speed threshold to analyze the game running demands in soccer players using GPS technology. Retos: nuevas tendencias en educación física, deporte y recreación; 32: 130-133 (2017)

26. R. K. Yin. Qualitative research from start to finish. New York, NY, USA: The Guilford Press (2015)

27. B. Yazan. Three approaches to case study methods in education: Yin, Merriam, and Stake. The Qualitative Report, 20(2), 134-152. (2015)

28. B.G. Glaser, A.L. Strauss. Discovery of grounded theory: Strategies for qualitative research. Routledge. doi: 10.4324/9780203793206. (2017) 